\title{
Comparison of a Population of Eastern Red-backed Salamanders, Plethodon cinereus, under Native Dominant Wood Coverboards and Natural Cover Objects
}

\author{
JEAN-DAVID MOORE
}

Direction de la Recherche forestière, Forêt Québec, Ministère des Ressources naturelles et de la Faune, 2700 rue Einstein, Sainte-Foy, Québec G1P 3W8 Canada; e-mail: jean-david.moore@mrnf.gouv.qc.ca

Moore, Jean-David. 2009. Comparison of a population of Eastern Red-backed Salamanders, Plethodon cinereus, under native dominant wood coverboards and natural cover objects. Canadian Field-Naturalist 123(3): 210-214.

Artificial cover objects or coverboards have been widely used to study Eastern Red-backed Salamanders (Plethodon cinereus), a species that is very abundant in eastern North America and known to be one of the most common vertebrates within its geographical range. However, recent studies have suggested that potential bias may be associated with the use of coverboards compared with natural cover objects. In this study, age structure and body size (weight and snout-vent length, SVL) of $P$. cinereus found under coverboards and natural cover objects at Lake Clair (Quebec, Canada) were compared. Coverboards were made of Sugar Maple (Acer sacchrum), a native forest species dominant in the study area. In 2004 and 2005 , a total of 162 P. cinereus were found under coverboards, and 156 P. cinereus were found under natural cover objects. No significant difference in the age structure, mean weight, or SVL of $P$. cinereus was observed between the two sets. This study suggests that the type of coverboard used at Lake Clair is a good method to obtain an accurate index of $P$. cinereus population demographics, and is similar to that expected under natural cover objects. This technique should help to establish a standard protocol that could better allow direct comparisons among studies in the future. However, more studies are needed to explain the high proportion of adult specimens found under both cover types at Lake Clair relative to studies in other regions in North America.

Key Words: Eastern Red-backed Salamander, Plethodon cinereus, capture comparison, wood coverboards, natural cover objects, northern hardwoods, Lake Clair, Lac Clair, Quebec.

The Eastern Red-backed Salamander (Plethodon cinereus) is a terrestrial species widely distributed in forests of eastern North America (Conant and Collins 1991). In many of these ecosystems, $P$. cinereus is probably the most abundant salamander (Burton and Likens 1975; Jaeger 1980) and terrestrial vertebrate (Burton and Likens 1975). Moreover, this salamander may play an important role within the food web of these forests, where they are consumers of small invertebrates (Burton and Likens 1975; Wyman 1998). In recent decades, many studies have demonstrated that these salamanders may also be good indicators of forest disturbance (deMaynadier and Hunter 1995; Patrick et al. 2006) and of changes in forest microhabitats (Wyman and Hawksley-Lescault 1987; deMaynadier and Hunter 1998).

Artificial cover objects or coverboards have been widely used to sample $P$. cinereus (cf. Moore 2005 for a review) because they may simulate downed woody debris, an important habitat component for $P$. cinereus (Heatwole 1962; Test and Heatwole 1962; Grover 1998). Other advantages of this technique include reduced habitat disturbance, modest time and labour costs, standardization of the number and area of cover items, and little between-observer variability (Fellers and Drost 1994). However, few studies have investigated how sampling using coverboards equates to population demographics found under natural cover objects (Monti et al. 2000; Marsh and Goicochea 2003). Marsh and Goicochea (2003) suggested that potential bias among size classes of $P$. cinereus may be associated with the use of coverboards as compared to natural cover objects.

Previous studies in the Lake Clair watershed show that using native dominant wood species as coverboards is a very efficient technique to sample $P$. cinereus (Moore 2005). However, whether sampling using coverboards represents the true population at Lake Clair is unknown. The objective of this study was to try and answer this question by comparing population demographics (snout-vent length (SVL), weight, and age structure) of $P$. cinereus found under coverboards and natural cover objects in this ecosystem.

\section{Study Site}

The Lake Clair watershed ( $226 \mathrm{ha}, 46^{\circ} 57^{\prime} \mathrm{N}, 71^{\circ} 40^{\prime} \mathrm{W}$, elevation 270 to $390 \mathrm{~m}$ ) is located approximately $50 \mathrm{~km}$ northwest of Québec City, Quebec, Canada. Forest vegetation is dominated by uneven-aged deciduous species $(83 \%)$ with small patches of conifers (17\%). The deciduous area is composed of Sugar Maple (Acer saccharum Marsh.), Yellow Birch (Betula alleghaniensis Britton), and American Beech (Fagus grandifolia Ehrh.). The coniferous area includes principally Balsam Fir (Abies balsamea (L.) Mill.) and a much lower proportion of Red Spruce (Picea rubens Sarg.) and White Birch (Betula papyrifera Marsh.). Soils are classified as Orthic Ferro-Humic Podzol (Canada 
Soil Survey Committee 1998) or Typic Haplorthod (Soil Survey Staff 1998). The humus is a mor-moder type, and the surface deposit is a very acidic and stony glacial till derived from granitic gneiss bedrock. Average slope is approximately $10 \%$. Mean annual temperature is $3.4^{\circ} \mathrm{C}$ and annual precipitation is $1300 \mathrm{~mm}$.

\section{Materials and Methods \\ Sampling}

In September 2001, 13 transects of 100 or $200 \mathrm{~m}$ in length were established around the lake, perpendicular to the lake shore, with a minimum distance of $300 \mathrm{~m}$ between each transect. Coverboards were placed along each of these transects at predetermined intervals from the lakeshore $(0,5,10,20,40,60,100$, $150,200 \mathrm{~m})$. Boards were placed on the transect line, on the nearest upper-soil surface available. A total of 104 coverboards were placed during this operation. Coverboards were constructed from radial sections of Sugar Maple trunks. Boards were $4 \mathrm{~cm}$ thick; the diameter varied from 36 to $42 \mathrm{~cm}$ and the surface area varied from 855 to $1400 \mathrm{~cm}^{2}$.

Coverboards were checked during daylight hours, on a single rainless day (sunny or cloudy), four times in each of 2002, 2003, 2004, and 2005, from the end of May through mid-October. Only the 2004 and 2005 sampling years were used in this study. Natural cover objects were checked in 2004 and 2005. They consisted of woody debris of different sizes, species (Sugar Maple, Yellow Birch, American Beech, Balsam Fir, Red Spruce), and degree of decomposition (solid wood to very decayed). Also, bark pieces on the soil surface, mainly from Sugar Maple trees, were checked. The surface area of 55 woody debris and bark pieces was measured. They varied in size from 5 to $55 \mathrm{~cm}$ in width, 10 to $400 \mathrm{~cm}$ in length, and 45 to $13700 \mathrm{~cm}^{2}$ in surface area.

Salamanders were first sampled under coverboards. To generate a relatively similar sampling size for both sampling methods (for comparison purposes), when $P$. cinereus was found under a coverboard, natural cover objects in a parallel area situated $15 \mathrm{~m}$ from the coverboard finding were checked for salamanders, based on how many salamanders were found under coverboards. Parallel sampling was done the same day or within a few days. Sampling effort was not noted, but it did not exceed 10 minutes for each parallel sampling.

Snout-vent length was measured in the field to the nearest $0.1 \mathrm{~mm}$ using a dial calliper. Salamanders were weighed to the nearest $0.1 \mathrm{~g}$ with a $10 \mathrm{~g}$ Pesola ${ }^{\circledR}$ spring scale. This type of balance has been widely used in other studies and monitoring programs to weigh lightweight salamanders (Anonymous 1999*; Gustafson et al. 2001; Zorn et al. 2004*; Moore 2005; Crawford and Semlitsch 2008). No attempt was made to deter- mine the gender of animals. Salamanders were handled in a transparent plastic bag that had previously been sprayed with lake water. After measurement, salamanders were immediately released at the edge of the coverboard or natural cover object under which they had been found. More information on methods can be found in Moore (2005).

\section{Stage classes used}

Many age structures have been proposed to categorize P. cinereus populations (Blanchard 1928; Test and Binghan 1948; Heatwole and Heatwole 1962; Sayler 1966; Bonin et al. 1999; Marsh and Goicochea 2003; Leclair et al. 2006). The structure proposed by Marsh and Goicochea (2003) in Virginia was used in this study (young of the year $(<25 \mathrm{~mm})$, juvenile (25-32 mm), and adult $(>32 \mathrm{~mm})$. It appeared to provide the best fit for the $P$. cinereus population at Lake Clair, as eggs were observed in some female $P$. cinereus of approximately $32 \mathrm{~mm}$ (SVL), indicating sexual maturity at this length. Moreover, this age structure is very similar to that which Bonin et al. (1999) used to study $P$. cinereus in southern Quebec.

\section{Statistics}

Analysis of variance (ANOVA) was employed to compare surface areas of natural cover objects and coverboards and to compare body parameters of the three developmental stage classes (adult, juvenile, and young of the year) of $P$. cinereus found under the two types of covers. The GML model procedure in SAS (SAS Institute 2008) was used. Frequency analysis was performed to compare the number of $P$. cinereus in each stage class found under natural cover objects and coverboards. Also, correlation analysis was used to evaluate the relationship between the surface areas of coverboards and natural cover objects and the three characteristics of $P$. cinereus (weight, snout-vent length, and total length). Outlier analysis and normality of the data were assessed with residuals.

\section{Results}

A total of $162 P$. cinereus (four of the lead colour phase) were found under coverboards in 2004 and 2005. Weight of salamanders varied from 0.05 to $1.60 \mathrm{~g}$, and SVL varied from 11.66 to $48.55 \mathrm{~mm}$. Mean weight and mean SVL were $0.86 \mathrm{~g}$ and $39.30 \mathrm{~mm}$, respectively. During the same period, 156 P. cinereus (eight of the lead phase) were found under natural cover objects. Weight of salamanders varied from 0.05 to $1.85 \mathrm{~g}$, and SVL varied from 17.81 to $49.92 \mathrm{~mm}$. Mean weight and mean SVL were $0.88 \mathrm{~g}$ and $40.47 \mathrm{~mm}$, respectively.

Year-to-year variations were noted, all cover types combined, for the weight of all the stage classes combined and for adults only $(P \leq 0.032)$, but not for juveniles and young of the year $(P>0.413)$. However, no interaction between the year and cover type was detected for weight and SVL for all the stage classes 
combined and each of the stage classes $(P>0.072)$. Also, no year-to-year variation was noted for SVL $(P>0.440)$.

Differences between mean surface areas $( \pm$ standard error) of coverboards $\left(1130 \pm 157 \mathrm{~cm}^{2}\right)$ and mean surface areas of natural cover objects $\left(2562 \pm 220 \mathrm{~cm}^{2}\right)$ were highly significant $(P<0.001)$. However, no relationship was found between the surface area of coverboards and natural cover objects and the weight $(P \geq 0.115)$ and SVL $(P \geq 0.455)$ of $P$. cinereus during the two-year sampling period. The effect of year $(P \geq 0.061)$ and season $(P \geq 0.098)$ was also not significant.

Over the two-year sampling period, only a slight difference in mean SVL for all $P$. cinereus (all stage classes combined) was detected between the two cover types (Table $1 ; P=0.043$ ). However, this difference was very small (the SVL of salamanders under coverboards was $39.22 \mathrm{~mm}$, and the SVL of salamanders under natural cover objects was $40.44 \mathrm{~mm}$ ). No significant difference was observed within stage classes $(P \geq 0.074)$ or mean weight $(P \geq 0.39)$ between $P$. cinereus found under coverboards and salamanders found under natural cover objects (Table 1).

The proportions of $P$. cinereus with cut tails captured under coverboards $(4.9 \%, 8$ out of 162) and natural cover objects $(3.2 \%, 5$ out of 156$)$ were not significantly different $(P=0.819)$.

\section{Discussion}

\section{Salamander size versus cover size}

No relationship was found between the surface area of the two board types and the sizes of $P$. cinereus, despite the wide range in the surface area of natural cover objects (45 to $13700 \mathrm{~cm}^{2}$ ). Previous study in 2002 and 2003 in the same experimental design also shows the same pattern for the coverboards (Moore 2005). Also, similar results were found in Virginia (Faragher and Jaeger 1997: coverboard surface area range of 10 to $5000 \mathrm{~cm}^{2}$; Gabor 1995: coverboard surface area $>50 \mathrm{~cm}^{2}$ ). Other studies found, however, that size or weight of $P$. cinereus was correlated with cover item size (Mathis 1990; Moore et al. 2000); in these cases, cover size effect was attributed to seasonal influence. This was, however, not the case at Lake Clair.

\section{Salamander size versus cover type}

Over the two-year sampling period, results of this study show that no important difference exists between the weight and SVL of salamanders found under coverboards and natural cover objects, even though the mean surface area of the natural cover objects was more than double that of the coverboards. These results are similar to several studies which found that salamanders found under coverboards were similar in size to those found under natural cover objects (Monti et al. 2000; Houze and Chandler 2002). However, they differ from the results of Marsh and Goicochea (2003), who reported that in a mature hardwood forest of Vir-

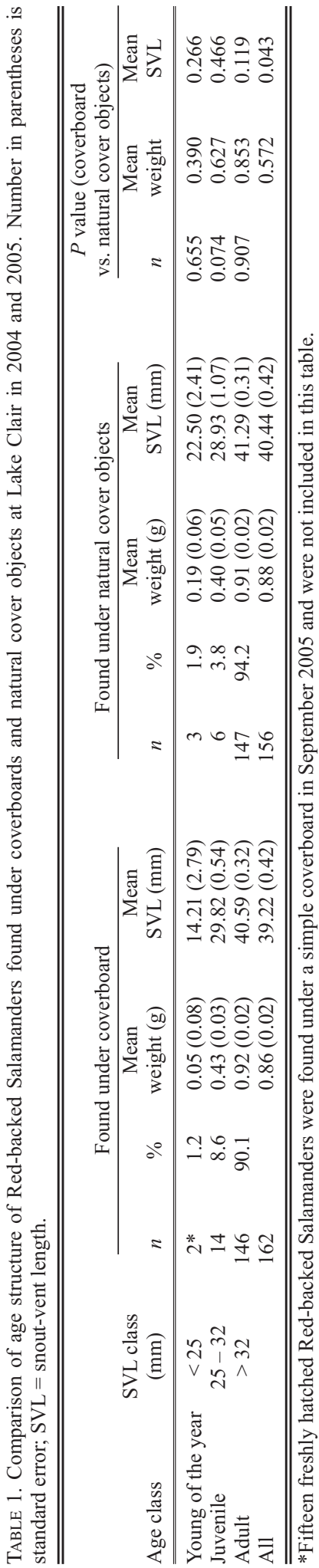


ginia, there were more adult and fewer juvenile and hatchling $P$. cinereus under coverboards than under natural cover objects.

It may be possible to account for the difference observed between the two studies. First, relatively high variability exists between the spring and fall 2001 surveys in Marsh and Goicochea's (2003) study. For example, fewer juvenile $P$. cinereus were captured in the spring survey from under coverboards than under natural cover objects (27 vs. 58), but more juveniles were captured in the fall from under coverboards than under natural cover objects (22 vs. 11). Second, many differences exist between the materials and methods of the two studies (e.g., age of coverboard, type of natural cover objects and coverboards, humus type, sampling period, sampling time). This last point highlights the need to establish standard protocols that could better allow direct comparisons of $P$. cinereus data among studies in the future, regardless of where they are conducted.

\section{Adult proportion}

The mean adult proportion for both cover types was relatively high at Lake Clair $(92 \%, 293$ out of 318; Table 1) compared with other studies (Blanchard 1928: 70\%, > $32 \mathrm{~mm}$; Test and Bingham 1948: 49\%, $\geq 34$ mm; Sayler 1966: 80\%, > 32 mm; Nagel 1977: 72\%, > $32 \mathrm{~mm}$; Faragher and Jaeger 1997: 74\%, $\geq 34 \mathrm{~mm}$; Bonin et al. 1999: 74\%, > $32 \mathrm{~mm}$; Marsh and Goicochea 2003: 46\%,> $32 \mathrm{~mm}$ ). Marsh and Goicochea (2003) proposed some hypotheses to explain the greater proportion of adults under coverboards than under natural cover objects. For instance, they mention that (1) adults may prefer coverboards to natural cover objects and (2) adults may be more evenly dispersed than juveniles and may move into the new habitat represented by coverboards. This would not explain the high proportion of adults under both wooden cover types in the Lake Clair watershed. A possible explanation for this phenomenon could be low recruitment of $P$. cinereus at Lake Clair compared to other studies.

Another possible explanation is that juvenile $P$. cinereus could be more abundant in the forest floor of this forest ecosystem, where abundant leaf litter is present (given the absence of foraging and litter-consuming earthworms). In northwestern Pennsylvania, Moore et al. (2000) found that the size and weight of $P$. cinereus were significantly greater for individuals captured under rock and downed wood than in leaf litter. In other studies, authors suggested that adult $P$. cinereus may tolerate the presence of juveniles in their territories (Jaeger et al. 1995; Faragher and Jaeger 1997). Also, Jaeger et al. (1995) observed territoriality in adult $P$. cinereus but not in juveniles. In this context, more studies will be needed to explain the high proportion of adult $P$. cinereus at Lake Clair.

\section{Predation or conspecific aggression}

The proportion of $P$. cinereus that had a cut tail was similar for both cover types (coverboard 4.9\%; natu- ral cover objects 3.2\%). These proportions are lower than those found in other parts of Quebec, where Bonin et al. (1999) reported a cut tail rate between 0 and $35 \%$ (mean $=9.8 \%$ ) among the 27 forests studied. These authors also found that cut tail rate was inversely correlated with the amount of woody debris on the forest floor. This suggests that the forest floor habitat of the Lake Clair watershed provides sufficient shelter for salamanders to allow P. cinereus to avoid important conspecific aggression (Jaeger 1981) or predation. Sugar Maple has been declining for more than a decade in the Lake Clair watershed (Duchesne et al. 2003), resulting in a relatively large volume of woody debris on the forest floor. The abundance of leaf litter in this ecosystem may also play a role by providing shelter during the displacement or dispersal of salamanders.

\section{Conclusion}

This study suggests that the type of coverboard (made of wood from a native dominant forest species) used at Lake Clair is a good method to obtain indices of $P$. cinereus population demographics similar to those of salamanders found under natural cover objects. This technique should help to establish a standard protocol that could better allow direct comparisons among studies in the future, regardless of where they are conducted.

\section{Documents Cited (marked * in text)}

Anonymous. 1999. Inventory methods for plethodontid salamanders. http://www.llbc.leg.bc.ca/public/PubDocs/bc docs/330771/psalm.pdf

Zorn, P., V. Blazeski, and B. Craig. 2004. Joint EMAN / Parks Canada National Monitoring Protocol for Plethodontid Salamanders. http://www.eman-rese.ca/eman/eco tools/protocols/terrestrial/salamanders/salamander.pdf.

\section{Literature Cited}

Blanchard, F. N. 1928. Topics from the life history and habits of the Red-backed Salamander in southern Michigan. American Naturalist 62: 156-164.

Bonin, J., J.-F. Desroches, M. Ouellet, and A. Leduc. 1999. Les forêts anciennes : refuges pour les salamandres. Le Naturaliste canadien 123: 13-18.

Burton, T. M., and L. E. Likens. 1975. Salamander populations and biomass in the Hubbard Brooks Experimental Forest, New Hampshire. Copeia 1975: 541-546.

Canada Soil Survey Committee. 1998. The Canadian System of Soil Classification. Publication 1646. 3rd edition. Ottawa, Ontario.

Conant, R., and J. T. Collins. 1991. A field guide to reptiles and amphibians: eastern and central North America. 3rd ed. Houghton Mifflin Co., Boston, Massachusetts, USA. 450 pages.

Crawford, J. A., and R. D. Semlitsch. 2008. Abiotic factors influencing abundance and microhabitat use of stream salamanders in southern Appalachian forests. Forest Ecology and Management 255: 1841-1847.

deMaynadier, M. L., and P. G. Hunter. 1995. The relationship between forest management and amphibian ecology: a review of the North American literature. Environmental Review 3: 230-261. 
deMaynadier, M. L., and P. G. Hunter. 1998. Effects of silvicultural edges on the distribution and abundance of amphibians in Maine. Conservation Biology 12: 340-352.

Duchesne, L., R. Ouimet, and C. Morneau. 2003. Assessment of Sugar Maple health based on basal area growth pattern. Canadian Journal of Forest Research 33: 20742080.

Faragher, R. G., and R. Jaeger. 1997. Distributions of adult and juvenile Red-backed Salamanders: testing new hypotheses regarding territoriality. Copeia: 410-414.

Fellers, G. M., and C. A. Drost. 1994. Sampling with artificial cover. Pages 147-150 in Measuring and monitoring biological diversity: standard method for amphibians. Edited by W. R. Heyer, M. A. Donnelly, R. W. McDiarmid, L. C. Hayek, and M. S. Foster. Smithsonian Institution Press, Washington, D.C.

Gabor, C. R. 1995. Correlational test of Mathis' hypothesis that bigger salamanders have better territories. Copeia 1995: 729-735.

Grover, M. C. 1998. Influence of cover and moisture on abundances of the terrestrial salamanders Plethodon cinereus and Plethodon glutinosus. Journal of Herpetology 32: 489-497.

Gustafson E. J., N. L. Murphy, and T. R. Crow. 2001. Using a GIS model to assess terrestrial salamander response to alternative forest management plans. Journal of Environmental Management 63: 281-92.

Heatwole, H. 1962. Environmental factors influencing local distribution and activity of the salamander Plethodon cinereus. Ecology 43: 460-472.

Heatwole, H., and A. Heatwole. 1962. Weight-length curve of the salamander Plethodon cinereus. Journal of the Ohio Herpetological Society 3: 37-39.

Houze, C. M., and C. R. Chandler. 2002. Evaluation of coverboards for sampling terrestrial salamanders in South Georgia. Journal of Herpetology 36: 75-81.

Jaeger, R. G. 1980. Microhabitats of a terrestrial forest salamander. Copeia 2: 265-268.

Jaeger, R. G. 1981. Dear enemy recognition and costs of aggression between salamanders. American Naturalist 117: 962-974.

Jaeger, R. G., J. A. Wicknick, M. R. Griffis, and C. D. Anthony. 1995. Socioecology of a terrestrial salamander: juveniles enter adult territories during stressful foraging periods. Ecology 76: 533-543.

Leclair, M. H., M. Levasseur, and R. Leclair. 2006. Lifehistory traits of Plethodon cinereus in the northern parts of its range: variations in population structure, age and growth. Herpetologica 62: 265-282.
Marsh, D. M., and M. A. Goicochea. 2003. Monitoring terrestrial salamanders: biases caused by intense sampling and choice of cover objects. Journal of Herpetology 37 : 460-466.

Mathis, A. 1990. Territoriality in a terrestrial salamander: the influence of resource quality and body size. Behavior 112: 162-174.

Monti, L., M. Hunter, and J. Witham. 2000. An evaluation of the artificial cover object (ACO) method for monitoring populations of the Redback Salamander Plethodon cinereus. Journal of Herpetology 34: 624-629.

Moore, J.-D. 2005. Use of native dominant wood as a new coverboard type for monitoring eastern Red-backed Salamanders. Herpetological Review 36: 268-271.

Moore, A. L., C. E. Williams, T. H. Martin, and W. J. Moriarity. 2000. Influence of season, geomorphic surface and cover item on capture, size and weight of Desmognathus ochrophaeus and Plethodon cinereus in Allegheny plateau riparian forests. American Midland Naturalist 145: $39-45$.

Nagel, J. W. 1977. Life history of the Red-backed Salamander, Plethodon cinereus, in northeastern Tennessee. Herpetologica 33: 13-18.

Patrick, D. A., H. L. Hunter Jr., and A. J. K. Calhoum. 2006. Effects of experimental forestry treatments on a Maine amphibian community. Forest Ecology and Management 234: 323-332.

SAS Institute Inc. 2008. SAS/STAT User's Guide, Version 9. SAS Institute Inc., Cary, N.C.

Sayler, A. 1966. The reproductive ecology of the Red-backed Salamander, Plethodon cinereus, in Maryland. Copeia 1966: 183-193.

Soil Survey Staff. 1998. Keys to soil taxonomy. 8th ed. USDA. Natural Resources Conservation Service, Washington, D.C.

Test, F. H., and B. A. Bingham. 1948. Census of a population of the Red-backed Salamander (Plethodon cinereus). American Midland Naturalist 39: 362-372.

Test, F. H., and H. Heatwole. 1962. Nesting sites of the Redbacked Salamander, Plethodon cinereus, in Michigan. Copeia 1962: 2006-2007.

Wyman, R. L. 1998. Experimental assessment of salamanders as predators of detrital food webs: effects on invertebrates, decomposition and the carbon cycle. Biodiversity Conservation 7: 641-650.

Wyman, R. L., and D. S. Hawksley-Lescault. 1987. Soil acidity affects distribution, behavior and physiology of the salamander Plethodon cinereus. Ecology 68: 1819-1827.

Received 4 May 2009

Accepted 21 April 2010 